STUDI

FRANCESI

\section{Studi Francesi}

Rivista quadrimestrale fondata da Franco Simone

170 (LVII | II) | 2013

Varia

\title{
Hanno Wijsman, Luxury bound. Illustrated Manuscript Production and Noble and Princely Book Ownership in the Burgundian Netherlands (1400-1550)
}

\section{Maria Colombo Timelli}

\section{(2) OpenEdition \\ Journals}

Édition électronique

URL : https://journals.openedition.org/studifrancesi/3014

DOI : 10.4000/studifrancesi.3014

ISSN : 2427-5856

Éditeur

Rosenberg \& Sellier

Édition imprimée

Date de publication : 1 juillet 2013

Pagination : 433-434

ISSN : 0039-2944

\section{Référence électronique}

Maria Colombo Timelli, « Hanno Wijsman, Luxury bound. Illustrated Manuscript Production and Noble and Princely Book Ownership in the Burgundian Netherlands (1400-1550) », Studi Francesi [En ligne], 170 (LVII I II) | 2013, mis en ligne le 30 novembre 2015, consulté le 02 février 2023. URL : http://

journals.openedition.org/studifrancesi/3014; DOI : https://doi.org/10.4000/studifrancesi.3014

Ce document a été généré automatiquement le 2 février 2023.

\section{c)}

Creative Commons - Attribution - Pas d'Utilisation Commerciale - Pas de Modification 4.0 International - CC BY-NC-ND 4.0

https://creativecommons.org/licenses/by-nc-nd/4.0/ 


\title{
Hanno Wijsman, Luxury bound. Illustrated Manuscript Production and Noble and Princely Book Ownership in the Burgundian Netherlands (1400-1550)
}

\author{
Maria Colombo Timelli
}

\section{RÉFÉRENCE}

HANNO WIJSMAN, Luxury bound. Illustrated Manuscript Production and Noble and Princely Book Ownership in the Burgundian Netherlands (1400-1550), Turnhout, Brepols, 2010, pp. 717.

1 Ce gros ouvrage constitue la version révisée et mise à jour de la thèse de doctorat que H.W. a défendue en 2003 à l'Université de Leiden. Il aborde trois sujets majeurs: la relation entre production et possession de manuscrits illustrés entre 1400 et 1550 , la formation de collections, le rapport entre livre manuscrit et livre imprimé à une époque caractérisée par une double circulation qui n'a cependant touché que marginalement les grandes bibliothèques princières. La matière, on s'en doute, est très abondante; elle est organisée en trois parties.

La première, Production, a pour but de présenter le corpus retenu, les données quantitatives permettant de reconnaître les tendances qui régissent la production de manuscrits illustrés dans les Pays-Bas bourguignons. Le premier chapitre (A corpus of late-medieval illustrated manuscripts from the Netherlands, pp. 15-36) définit les critères de composition et d'interprétation de ce corpus, formé par 3620 manuscrits enluminés produits entre 1400 et 1550 dans la vaste région correspondant aujourd'hui aux PaysBas, Belgique, Luxembourg, Nord-Pas-de-Calais (cf. Appendice A); ce chiffre représenterait en gros, selon H.W., un cinquième de la production originale. On passe 
ensuite à des analyses quantitatives: le chapitre 2 (Chronology and geography, pp. 37-79) vérifie la distribution de cette production par décennies (cf. Appendice B) et par aires géographiques (cf. carte, fig. 1), en concentrant l'attention sur les centres de copie et d'illustration, et, à partir des années 1470 , les ateliers d'imprimerie. Le troisième chapitre (Texts and languages, pp. 81-104) offre des classements par genres (les dix genres retenus sont illustrés et justifiés dans l'Appendice C) et par langues (français, latin, néerlandais surtout); les différentes données sont croisées dans de nombreux tableaux qui permettent une approche plus complexe et une observation approfondie des phénomènes en jeu. Le chapitre 4 (Numbers and sizes, pp. 105-121) concerne le nombre et la taille des enluminures par rapport au genre, au matériau, aux dimensions des manuscrits, avec un regard particulier pour les livres d'heures.

3 La deuxième partie, Ownership, a pour objet l'examen des librairies de l'époque. Après avoir discuté les questions rattachées à la commande de manuscrits enluminés et de textes (chapitre 5, Late-medieval manuscript ownership, pp. 125-143), H.W. présente la collection des ducs de Bourgogne selon une approche quantitative fondée sur des informations matérielles: inventaires, genres, supports, langues, illustrations, reliures (chapitre 6, The Burgundian Library, pp. 145-170), puis les sous-collections que l'on peut rattacher à la bibliophilie féminine des plus grandes dames de cette époque: Marguerite de Male, Marguerite de Bavière, Isabelle de Portugal, Isabelle de Bourbon, Marguerite d'York, Marie de Bourgogne, Marguerite d'Autriche (chapitre 7, Sub-collections for women and children, pp. 171-217), pour en arriver à la collection réunie par Philippe le Bon, dont le règne est divisé par H.W. en deux phases, avant et après ca 1445; sont particulièrement examinés la commande de chroniques, les livres présents en plusieurs exemplaires dans sa «librairie», l'absence de minutes (chapitre 8, The manuscripts of Philip the Good, pp. 219-255). Les deux derniers chapitres de cette section offrent de vastes panoramiques des collections réunies par les représentants de la plus haute noblesse (chapitre 9, Book ownership among the high nobulity: a survey, pp. 257-480: rappelons entre autres Antoine de Bourgogne et Louis de la Gruuthuse, les Clèves, Créquy, Croÿ, Lalaing, Lannoy, Luxembourg, Nassau, Wavrin) et par les gens de cour, officiers, membres du patriciat urbain et petite noblesse (chapitre 10, Book ownership among the new men at court, pp. 481-499).

Deux chapitres composent la dernière partie, Convergences, et peuvent être considérés comme le bilan de l'ensemble. Le chapitre 11 (Noble and princely book ownership in the Burgundian Netherlands, pp. 503-553) récupère les données réunies dans la seconde partie pour éclaircir d'autres aspects, notamment le rapport entre les commandes de manuscrits illustrés et l'âge des commanditaires, les collections réunies par les membres de l'ordre chevaleresque de la Toison d'or, le rapport entre production de livres enluminés et lecture, les ateliers de copie et d'enluminure, le réseau de nobles hommes et femmes - collectionneurs autour de la cour des ducs, le cas particulier des livres d'heures. Le chapitre 12 (The manuscript market, pp. 555-568) représente une sorte de conclusion générale.

5 Outre les appendices déjà cités, on trouvera en annexe: une liste de 53 manuscrits illustrés par le Maître aux mains volubiles, actif à Bruges entre 1470 et 1480 (pp. 579-586), l'édition d'une liste de 86 titres d'ouvrages qui apparaît au f. 200r-v du ms Bruxelles, KBR, 10437-40 (pp. 587-596), des diagrammes statistiques traduisant visuellement quelques-unes des tables précédentes (pp. 597-605). La bibliographie occupe une quarantaine de pages en petits caractères (pp. 607-646). Quarante 
illustrations, malheureusement en noir et blanc, donnent une idée de l'iconographie des manuscrits commentés (pp. 647-681). S'ajoutent encore deux index, des manuscrits (pp. 683-695) et des noms (pp. 697-717); au risque de paraître injuste devant la masse des informations offertes par H.W., signalons celle qui nous paraît une regrettable lacune, à savoir l'absence d'un index des œuvres citées.

6 Ce livre constitue une mine de renseignements et de réflexions qui sera appréciée par de nombreux spécialistes du Moyen Âge tardif. Ayant essayé de le consulter comme une sorte d'encyclopédie, nous avons néanmoins découvert quelques faiblesses, par exemple p. 438, où il est dit qu'une copie de la Vie de saint Furcy, œuvre de Jean Miélot dont l'édition critique est en préparation par Elisabetta Barale (thèse de doctorat à l'Université de Turin, sous la direction de Paola Cifarelli) se trouve dans le ms. fr. 17001 de la BnF: ceci est malheureusement faux, puisque ce ms. ne contient qu'un arbre généalogique du Saint (f. 96r) et quelques enluminures qui le concernent (f. 3r, où ses parents sont représentés à l'intérieur d'un grand $\mathrm{C}$ historié; $\mathrm{f}$. $3 \mathrm{v}$, où son portrait est enchâssé dans un grand $\mathrm{D}$; et $\mathrm{f}$. $4 \mathrm{v}$, où ses deux frères, saint Foillain et saint Ultain trouvent place à l'intérieur des boucles d'un $\mathrm{M}$ ), alors que la Vie est transmise par deux autres manuscrits, l'un à Vienne (ÖNB, Series Nova 2731), l'autre en mains privées. Mes compétences étant des plus limitées, je ne saurais me permettre de juger un si gros ouvrage à l'aune d'une petite erreur; je ne la signale que comme une mise en garde au lecteur, une invitation à utiliser certaines données avec prudence, et éventuellement à les vérifier.

7 En conclusion, nous avons là un livre aussi luxueux que son objet, imprimé sur papier couché, un véritable cadeau pour les passionnés de cette période magnifique que fut la Bourgogne des ducs Valois. 